\section{Agricultural pesticide exposure and chronic kidney disease: new findings and more questions}

\author{
Geoffrey M Calvert
}

The vital importance of agriculture is well-recognised, as is the usefulness of pesticides in increasing agricultural yields and reducing spoilage rates. The usefulness of pesticides in mitigating diseasecarrying pests (eg, mosquitos) is also well known. However, there are also risks associated with pesticide use. In addition to causing acute poisoning, ${ }^{1}$ they are also associated with increased cancer risks, ${ }^{2}$ among other diseases. A paper by Lebov and colleagues ${ }^{3}$ provides evidence for another potential risk associated with pesticides, that is, end-stage renal disease (ESRD). To our knowledge, this is the first report using the United States Renal Data System (USRDS) to assess the association between pesticide exposure and ESRD.

Currently, there is little literature available on the nephrotoxic effects of pesticides. The little research that does exist comes from animal studies and case reports of pesticide-poisoned individuals. Fortunately, our understanding of the role of occupational exposures, including pesticides, on ESRD development in humans is growing. An important tool supporting the growth of this understanding is the USRDS. ${ }^{4}$ Since the US government provides healthcare coverage, under Medicare, for all patients with ESRD and because these ESRD claims data are comprehensively captured by USRDS, USRDS represents a nearly complete national disease registry in the USA. Furthermore, because there is no other medical condition so covered by the US government, there is no other disease or injury in the USA that has such a nearly complete national registry. From the first use of USRDS to identify occupational exposures associated with ESRD, which in that initial case involved an exploration of silica exposure, ${ }^{5}$ it has been used to identify ESRD associations with several other occupational exposures, including perchloroethylene, ${ }^{6}$ lead $^{7}$ and 1,1,1-trichloroethane. ${ }^{8}$ With the paper in this issue of OEM, USRDS has now been

Correspondence to Dr Geoffrey M Calvert, Division of Surveillance, Hazard Evaluations, and Field Studies, National Institute for Occupational Safety and Health, 1090 Tusculum Avenue, R-17, Cincinnati, $\mathrm{OH} 45226$, USA; jac6@cdc.gov used to assess the association between pesticide exposure and ESRD. ${ }^{3}$ Lebov et al matched data from the Agricultural Health Study (AHS), a very large prospective study of licensed pesticide applicators, with data from USRDS to identify cohort members with ESRD and to determine if the observed cases exceeded population rates. Although these authors provided reassuring findings of no increased ESRD risk in the overall cohort, they did find significantly increased ESRD risks and positive exposure-response trends, among pesticide applicators who mixed or applied one or more of six specific pesticides. These pesticides included five herbicides (alachlor, atrazine, metolachor, paraquat and pendimethalin) and the insecticide permethrin.

This paper by Lebov et al provides a substantial contribution to our understanding of the role of pesticides in ESRD risk. However, our enthusiasm must be tempered by the fact that this paper examined ESRD risk for only 39 pesticide active ingredients (in a pesticide product, the active ingredient is the chemical that kills or otherwise controls a pest). In contrast there are at least 1100 pesticide active ingredients currently sold in the USA, and over 2500 additional pesticide active ingredients that are no longer available for sale in the USA. ${ }^{9}$ As such, this study examined only a tiny fraction of pesticide active ingredients.

Of the six active ingredients found to be significantly related to ESRD, three are restricted use pesticides that can only be purchased and used by licensed pesticide applicators (ie, alachlor, atrazine and paraquat). The other three are available for sale to homeowners. Although Lebov et $a l^{3}$ did not examine the risk of ESRD from homeowner use, nor from the low level residues of these pesticides that may be present on fruits and vegetables consumed by the public, it is reassuring that workers in the lowest exposure category for these pesticides did not have a significantly increased ESRD risk.

Lebov et al considered only pesticide exposures that occurred before study enrolment, arguing that the latency from normal kidneys to ESRD can take decades. This analytic approach of only considering exposures that occurred decades earlier suggests primary interest in factors related to chronic kidney disease initiation. However, pesticides could also be responsible for ESRD progression. That is, pesticide exposure may cause accelerated worsening of already damaged renal function leading to more rapid ESRD development. An assessment of post-enrollment pesticide exposures may reveal whether pesticides are involved in chronic kidney disease initiation versus progression.

Do these six pesticides effect all types of ESRD? Unfortunately, this study did not address this question. There are several types of ESRD (ie, attributed causes of ESRD). The most common ESRD types are those caused by diabetes or hypertension (ie, diabetic ESRD and hypertensive ESRD). ${ }^{4}$ Other common causes/types of ESRD are glomerulonephritis and interstitial nephritis. Knowledge of the cause of ESRD can be helpful when targeting interventions. For example, if the AHS investigators had determined that the increased ESRD risk associated with a given pesticide was driven by diabetic ESRD, measures could be taken to prevent progression of diabetic kidney damage (eg, through improved glucose and blood pressure control, and dietary protein restriction). Interestingly, of the six pesticides found to be associated with ESRD, previous analyses of AHS data found that alachlor was also associated with an increased risk of incident diabetes. ${ }^{10}$ Knowing the type of ESRD may also assist the patient in obtaining workers' compensation benefits. For example, if it were determined that alachlor use is associated with diabetic ESRD risk, then the likelihood of being granted benefits would be bolstered whenever diabetic ESRD is diagnosed in any agricultural worker who often handled alachlor.

Lebov et al also found a significantly increased ESRD risk, and a positive exposure-response trend among those who sought medical care after any pesticide use (ie, this association was not restricted to the six pesticides mentioned earlier). Intriguingly, they did not find an association between ESRD and a history of physician-diagnosed pesticide poisoning. There are several reasons that could explain this discrepancy. First, sample sizes differed for these outcomes. Information on physician-diagnosed pesticide poisoning was available for less than half of AHS participants, whereas information on medical visits and hospitalisations due to pesticide use was available on all AHS participants included in this 
analysis. Second, because most healthcare professionals are not acquainted with the recognition of pesticide poisoning, and because the signs and symptoms of these poisonings are not pathognomonic, patients who seek medical care may not receive an accurate diagnosis. Furthermore, a pesticide poisoning diagnosis may not be made because laboratory diagnostic tests are rarely available, and healthcare professionals who are acquainted with pesticide poisoning may be reluctant to make such a diagnosis without confirmatory laboratory tests. Finally, because most cases of pesticide poisoning are self-limited and treated symptomatically, from a purely clinical perspective it may make little difference what diagnosis is assigned to the patient. However, attributing the illness to pesticide poisoning can place additional burdens on the physician. These burdens may include the need to report the case to public health authorities and the requirement to seek reimbursement from workers' compensation insurance companies which may lead to additional paperwork and payment delays.

It can be argued that agriculture is the first and oldest industry. Despite the ancient heritage of this industry, those employed in it continue to face elevated risks to their health and safety, many from exposures that are of relatively recent origin (eg, tractors and pesticides). ESRD may be another health risk facing workers employed in this industry.

Twitter Follow Geoffrey Calvert at @gmcalvert1

Competing interests None declared.

Provenance and peer review Commissioned; internally peer reviewed.

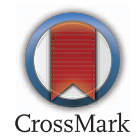

To cite Calvert GM. Occup Environ Med 2016;73:1-2.

Received 23 July 2015

Revised 13 August 2015

Accepted 2 September 2015

Published Online First 24 September 2015

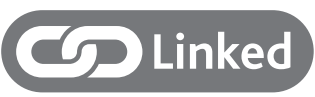

- http://dx.doi.org/10.1136/oemed-2014-102615

Occup Environ Med 2016;73:1-2.

doi:10.1136/oemed-2015-103132

\section{REFERENCES}

1 Calvert GM, Karnik J, Mehler L, et al. Acute pesticide poisoning among agricultural workers in the United States, 1998-2005. Am J Ind Med 2008;51:883-98.

2 Alavanja MCR, Ross MK, Bonner MR. Increased cancer burden among pesticide applicators and others due to pesticide exposure. CA Cancer I Clin 2013;63:120-42.
3 Lebov JF, Engel LS, Richardson D, et al. Pesticide use and risk of end-stage renal disease among licensed pesticide applicators in the Agricultural Health Study. Occup Environ Med 2016;73:3-12.

4 Saran R, Li Y, Robinson B, et al. US Renal Data System 2014 annual data report: epidemiology of kidney disease in the United States. Am I Kidney Dis 2015;66 (suppl 1):S1-306.

5 Calvert GM, Steenland K, Palu S. End stage renal disease (ESRD) among silica-exposed gold miners: a new method for assessing incidence among epidemiologic cohorts. JAMA 1997;277: 1219-23.

6 Calvert GM, Ruder A, Petersen MR. Mortality and end-stage renal disease (ESRD) incidence among dry cleaning workers. Occup Environ Med 2011;68:709-16.

7 Chowdhury R, Darrow L, McClellan W, et al. Incident ESRD among participants in a lead surveillance program. Am J Kidney Dis 2014; 64:25-31.

8 Radican L, Wartenberg D, Rhoads GG, et al. A retrospective occupational cohort study of end-stage renal disease in aircraft workers exposed to trichloroethylene and other hydrocarbons. J Occup Environ Med 2006;48:1-12.

9 USEPA. Pesticide product information system. Washington DC: US Environmental Protection Agency, 2015. http://www2.epa.gov/ingredientsused-pesticide-products/ppis-download-productinformation-data (accessed 23 Jul 2015).

10 Montgomery MP, Kamel F, Saldana TM, et al. Incident diabetes and pesticide exposure among licensed pesticide applicators: agricultural Health Study, 1993-2003. Am J Epidemiol 2008;167:1235-46. 